\title{
Influence de l'état physiologique sur l'homéostase énergétique après l'injection d'insuline chez la chevrette en gestation
}

\author{
M Lloret-Pujol, P Schmidely, P Bas, D Sauvant
}

\begin{abstract}
INRA, station de nutrition et alimentation de I'INA-PG,
\end{abstract} 16, rue Claude-Bernard, 75231 Paris cedex 05, France

De l'insuline est injectée dans la veine jugulaire $(0,12 \mathrm{Ul} / \mathrm{kg})$ à des chevrettes à $86(\mathrm{G1}, \mathrm{n}=5)$, $111(G 2, n=4)$ et $142(G 3, n=4)$ j de gestation et à 6 chevrettes non saillies (NG), du même âge que les chevrettes gestantes $(10,11$ et 12 mois). Des échantillons de sang sont prélevés avant l'injection (t0) et $5,10,15,20,35,49$ et $81 \mathrm{~min}$ après le début de l'injection afin de mesurer les teneurs plasmatiques en glucose (GLU), insuline (INS), $\beta$-hydroxy-butyrate (BHB) et acides gras non estérifiés (AGNE).

Les valeurs basales sont pour le GLU : 3,28, $3,36,2,85$ et $3,62 \mathrm{mM} /(S E M=0,08)$; pour l'INS : $17,23,14,58,10,25$ et $24,078 \mu \mathrm{U} / \mathrm{ml}(\mathrm{SEM}=$ $2,54)$; pour le BHB : $0,16,0,19,0,22$ et $0,15 \mathrm{mM}$ (SEM $=0,01$ ) et pour les AGNE : 113, 251, 494, $129 \mu \mathrm{M} / /(\mathrm{SEM}=38)$, respectivement pour les lots G1, G2, G3 et NG. La glycémie et l'insulinémie diminuent tandis que la concentration plasmatique des AGNE et du BHB augmente à mesure que la gestation avance, conformément aux résultats de Rémésy et Demigné (1976).

La corrélation $(r=-0,63)$ entre le paramètre Go (GLU à to) et le paramètre AO (AGNE à t0) permet de dégager 2 profils métaboliques extrêmes et opposés d'état énergétique : les animaux en état de mobilisation ( $A O$ élevé associé à Go faible) principalement constitués par les animaux de fin de gestation sont à opposer aux autres animaux, principalement des chevrettes non gestantes (AO faibles associés à GO élevés).

La courbe de disparition de l'insuline a été ajustée à un modèle non linéaire mono-compartimental : INS = INSO $\times e^{-k t}$. II n'y a pas de différences significatives entre les lots pour les para- mètres des ajustements. Leurs valeurs moyennes sont : INSO = $1225 \mu \mathrm{U} / \mathrm{(SE}=110)$ et $k=0,126$ min-1 $^{-1}$ (SE $\left.=0,005\right)$.

La chute maximale de glycémie après l'injection d'INS (G1) est positivement liée à la glycémie intiaie (Go) :

$$
\begin{aligned}
& \mathrm{G} 1=0,648( \pm 0,025) \times \mathrm{G} 0 \\
& \left(\mathrm{R}^{2}=0,48, P<0,01, n=19\right)
\end{aligned}
$$

La diminution maximale des AGNE (A1) obtenue à 15 min après injection et l'accroissement ultérieur (t15 à t49) des AGNE (A2) sont corrélés à leur niveau basal :

$$
\begin{aligned}
& A 1=0,372( \pm 0,051) \times A 0 \\
& \left(R^{2}=0,38, P<0,01, n=19\right) \\
& A 2=256,62( \pm 72,90)+( \pm 0,261) \times A 0 \\
& \left(R^{2}=0,36, P<0,01, n=19\right)
\end{aligned}
$$

Ceci traduit une sensibilité accrue de l'activité lipolytique du tissu adipeux à l'hypoglycémie lors de bilans énergétiques négatifs.

L'état métabolique initial semble être le paramètre prédominant de la réponse à l'INS ; le stade de gestation ne paraît pas avoir d'effet propre, au-delà du bilan énergétique négatif qu'il induit. Les résultats obtenus confirment l'insulino-résistance glucidique chez les animaux en statut énergétique défavorable ; il existe, en revanche, une réponse accrue du tissu adipeux à une stimulation lipolytique chez les mêmes animaux.

Rémésy C, Démigné (1976) Ann Rech Vét 7, 329-341 